\title{
Prognostic biomarkers of chronic diabetic macular edema treated with a fluocinolone acetonide intravitreal implant
}

Beatriz Oliveira Lopes ${ }^{1}$, Margarida Sena Brizido ${ }^{1}$, Florence Aerts ${ }^{1}$, Susana Morais Pina ${ }^{1}$, Pedro Santana Simoes ${ }^{1}$ and Margarida Isidoro Miranda ${ }^{1}$

${ }^{1}$ Department of Ophthalmology, Beatriz Angelo Hospital, Loures, Portugal

\begin{abstract}
Background: This study aimed to investigate retinal imaging biomarkers, such as disorganization of the retinal inner layers (DRIL) and/or ellipsoid zone (EZ) disruption by spectral domain optical coherence tomography $(\mathrm{SD}-\mathrm{OCT})$, and functional outcomes in eyes treated with $0.2 \mu \mathrm{g} /$ day of a fluocinolone acetonide intravitreal implant (FAc) after an insufficient response to previous treatments.

Methods: This was a retrospective comparative study of 18 eyes (15 patients) with persistent and/or recurrent diabetic macular edema (DME) treated with FAc. Eyes were divided according to the number of prior intravitreal treatments: group $1(\mathrm{n}=8)$ with $\leq 6$ injections (early switch) and group $2(\mathrm{n}=10)$ with $>6$ injections (late switch). Outcomes included percentage of eyes with DRIL and/or EZ disruption at baseline and analysis of the best corrected visual acuity (BCVA) using ETDRS letters, central macular thickness (CMT), DRIL, and EZ disruption at the last observation.

Results: Group 2 revealed a significantly higher percentage of DRIL and/or EZ disruption than group 1 $(P<0.05)$. At the last observation, group 1 revealed a higher percentage of eyes achieving vision stability/ improvement, gaining $\geq 15$ letters, and achieving $\geq 70$ letters $(P>0.05$ for all comparisons). The mean BCVA gain was 8.8 and 0.7 letters for groups 1 and $2(P=0.397)$. Both groups revealed a significant mean CMT reduction $(>20 \%$ reduction from the baseline value), without a significant statistical difference between them $(P=0.749)$. After treatment, most eyes from both groups showed resolution of DRIL and EZ disruption. Conclusions: Patients with DME presenting with a lower percentage of DRIL and/or EZ disruption at baseline had better functional outcomes, supporting the possible benefit of an early switch to FAc after insufficient response to previous treatments. Future randomized studies with a larger patient cohort are warranted to confirm our conclusions.
\end{abstract}

\section{KEYWORDS}

spectral domain optical coherence tomography, SD-OCT, diabetic macular edema, fluocinolone acetonide intravitreal implant, retina imaging biomarkers, disorganization of retinal inner layers, ellipsoid zone, prognostic biomarkers, central macular thickness

\section{INTRODUCTION}

Diabetic macular edema (DME) is the most common cause of blindness in patients with diabetic retinopathy [1]. The prevalence of DME has been reported to be $24.5 \%$ in patients with type II diabetes throughout a

Correspondence: Beatriz Nunes de Oliveira Lopes, MD. Av. Carlos Teixeira 3, 2674-514 Loures, Portugal. Email: bibas.oliveiralopes@gmail.com. ORCHID iD: https://orcid.org/0000-0002-9622-3692

How to cite this article: Lopes BO, Brizido MS, Aerts F, Pina SM, Simoes PS, Miranda MI. Prognostic biomarkers of chronic diabetic macular edema treated with a fluocinolone acetonide intravitreal implant. Med Hypothesis Discov Innov Ophthalmol. 2021 Summer; 10(2): 50-58. https://doi.org/10.51329/mehdiophthal1421

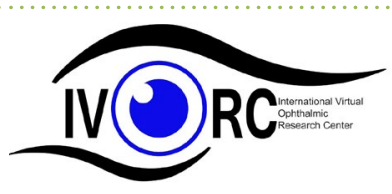

Copyright (C) Author(s). This is an open-access article distributed under the terms of the Creative Commons Attribution-NonCommercial 4.0 International License (http://creativecommons.org/licenses/by-nc/4.0/) which permits copy and redistribute the material just in noncommercial usages, provided the original work is properly cited. (c) (i) (s) 
10 -year period [2, 3]. Although hyperglycemia is considered a major risk factor for the development of DME, the exact mechanism of its pathogenesis remains unclear [4]. Hyperglycemia is responsible for the upregulation of inflammatory cytokines and growth factors such as interleukin (IL)-8, intercellular adhesion molecule 1 (ICAM1), and vascular endothelial growth factor (VEGF) [2, 4]. In its multifactorial nature, blood-retinal barrier dysfunction and inflammation may also play an important role $[1,4]$. Therefore, besides anti-VEGF therapy, which is considered the mainstay of DME treatment [5, 6], intravitreal corticosteroids, acting mainly against the inflammatory component of DME, are becoming increasingly important, particularly in chronic or persistent DME [7-9]. Indeed, DME presents with a widely variable response to anti-VEGF treatment [1]. Protocols T and I in the Diabetic Retinopathy Clinical Research Network (DRCR.net) show that nearly 40\% of eyes have persistent DME after six anti-VEGF monthly injections [10, 11].

The fluocinolone acetonide (FAc) implant is a non-biodegradable corticosteroid intravitreal implant that provides controlled and sustained drug release $(0.2 \mu \mathrm{g} /$ day $)$ during 3 years $[7,12,13]$. It can act against proinflammatory molecules such as VEGF-A, chemokines, and cytokines, reducing inflammation, angiogenesis, and vascular permeability $[7,13]$. In Europe, the FAc implant is indicated for the treatment of chronic DME, which is considered insufficiently responsive to available therapies $[3,7]$. Despite the higher risk of cataract formation and ocular hypertension associated with intravitreal corticosteroid agents [6], there are several benefits to using FAc implants. The benefits include fewer potential systemic side effects (from repeated anti-VEGF treatments), longer treatment intervals with reduction of treatment burden, and higher patient compliance [7, 8].

Spectral domain optical coherence tomography (SD-OCT), or athwart acquisition of high-resolution retinal anatomical images, offers a strong morpho-functional correlation that is particularly useful in DME management $[1,6]$.

Central retinal thickness (CMT) and other essential parameters, such as glycemic control, are considered insufficient and inadequate predictors of future visual acuity in patients with DME $[14,15]$. The identification of reliable visual surrogates and functional prognostic markers to select appropriate intravitreal treatments and determine future therapeutic responses is now fundamental and urgent. Recently, SD-OCT has become extremely important in this field, allowing evaluation of retinal integrity through prognostic biomarker analysis, such as disorganization of the retinal inner layers (DRIL) and ellipsoid zone (EZ) disruption [6, 14-17].

Chronicity may play an important role in the development and evolution of DRIL [18], since patients with chronic DME have a higher incidence of this macular biomarker [17, 18]. For that reason, hypothetically, treating patients earlier may lead to better functional results, once retinal integrity at the baseline seems to be directly correlated with visual outcomes $[17,19]$. However, as the pathogenesis of these biomarkers has been related to the inflammatory status of DME, treatment with FAc implants is particularly interesting in these eyes [17].

In eyes with chronic DME, we assessed the extent of DRIL and/or EZ disruption and compared these and functional outcomes following early or late switch to an FAc intravitreal implant.

\section{METHODS}

This non-interventional, retrospective, comparative, single-center study included 18 eyes from 15 patients with chronic DME (i.e., DME that persists or recurs despite treatment) [3]. The study was conducted at the Beatriz Ângelo Hospital, a tertiary referral center in Loures, Portugal. Patients were included if they had been treated with an FAc intravitreal implant according to the approved license in Europe and after providing informed consent. The study protocol was approved by the ethics committee of the institute (Health Ethical Committee of Beatriz Ângelo Hospital).

Two patient groups were devised based on the number of prior intravitreal treatments: group 1 included 8 eyes ( 7 patients) that had received $\leq 6$ prior intravitreal injections (early switch group; including anti-VEGF intravitreal injections such as bevacizumab [Avastin ${ }^{\circledast}$; Roche ${ }^{\bowtie}$, Grenzach, Germany], aflibercept [Eylea ${ }^{\circledR}$; Bayer Pharma $\mathrm{AG}^{\circledast}$, Leverkusen, Germany], and ranibizumab [Lucentis ; Novartis ${ }^{\circledast}$, Dublin, Ireland] or shortacting corticosteroid intravitreal injections such as a triamcinolone (Intracinol ${ }^{\circledR}$; Farmigea ${ }^{\circledR}$, Pisa, Italy) or a dexamethasone (Ozurdex ${ }^{\circledast}$; Allergan Inc ${ }^{\oplus}$, Dublin, Ireland) and group 2 included 10 eyes (8 patients) that had received $>6$ intravitreal injections (late switch group).

All patients underwent a complete ophthalmologic examination, which included best corrected visual acuity (BCVA), anterior and posterior segment slit-lamp evaluation (Takagi SM-70N, Takagi Seiko Co.,Ltd., Japan), and Goldmann applanation tonometry (AT 900R ${ }^{\oplus}$, Haag-Streit, Koeniz, Switzerland). BCVA was measured using the Early Treatment Diabetic Retinopathy Study (ETDRS) protocol at a 4-meters distance with an ETDRS chart and calculated in accordance with the ETDRS scoring method [20].

In all patients, SD-OCT was performed at each visit using Spectralis (Heidelberg Engineering; Heidelberg, 
Germany) in high resolution mode with 100 automatic real-time (ART) and $6 \times 6 \mathrm{~mm}$ macular map. Vertical and horizontal linear scans centered on the fovea were obtained. Two independent blinded graders analyzed the following parameters: CMT, DRIL, and EZ disruption. DRIL and EZ were obtained on the 1-mm diameter central region, and the patient's eyes were further classified according to the presence or absence of these imaging biomarkers. Foveal DRIL was defined as the inability to clearly distinguish the boundaries between the inner plexiform, inner nuclear, and outer plexiform layers $[14,15]$. EZ disruption was defined as the inability to identify the continuity of the inner segment-outer segment photoreceptor junction and loss of reflectivity in these layers [14].

The inclusion criteria were diagnosis of diabetes mellitus type 2; the presence of clinically significant DME (according to ETDRS guidelines), either persistent or recurrent; and treatment with an intravitreal FAc implant for at least 12 months. The exclusion criteria were macular edema secondary to causes other than DME; the presence of concurrent retinal diseases such as macular degeneration or retinal vein occlusion; high media opacities; any ophthalmic surgery in the last 6 months prior to the FAc implant; and uncontrolled glaucoma or any history of uveitis or other ophthalmic or systemic disease potentially affecting the results of the study.

Outcome measures included the percentage of eyes with DRIL and/or EZ disruption at baseline and analysis of the BCVA, CMT, and intraocular pressure (IOP) at baseline, months 1 and 3, and then quarterly thereafter until the last observation (mean \pm standard deviation [SD], $2.43 \pm 16.25$ months for group 1 and $12.94 \pm 15.00$ months for group 2). All outcomes were also analyzed at the last observation.

Descriptive statistics (means and absolute and relative frequencies) and inferential statistics were used. Statistical analysis was performed using IBM SPSS Statistics for Windows, version 25.0 (IBM Corp., Armonk, NY, USA). The paired sample Student's t-test was performed to compare parametric data between groups, and the Wilcoxon non-parametric test was used for comparisons of non-parametric values. The Shapiro-Wilk test was used when the variables did not present a normal distribution. Fisher's test was used when comparing groups in nominal type variables. The Yates Chi-square association test was also performed to compare the effect of treatment on OCT biomarkers between groups. The level of statistical significance was set at $P<0.05$.

\section{RESULTS}

This study included 18 eyes (15 patients) with persistent and/or recurrent DME treated with FAc. Eyes were divided according to the number of prior intravitreal treatments: group $1(n=8)$ with $\leq 6$ injections (early switch) and group $2(\mathrm{n}=10)$ with $>6$ injections (late switch). Table 1 shows the baseline demographics and ocular characteristics of the patients. At baseline, there were no significant differences in the mean age, mean duration of DME, and lens status (all $P>0.05$ ) between the two groups. The mean follow-up duration was similar for groups 1 and $2(P>0.05)$. The number of previous intravitreal injections was significantly $(P=0.001)$ lower in group $1(4.88 \pm 1.36)$ than in group $2(8.90 \pm 2.03)$ and reflected fewer $(P=0.001)$ intravitreal antiVEGF injections in group 1 than in group $2(3.88 \pm 1.33$ versus $7.80 \pm 1.81$, respectively) (Table 1$)$.

Table 1. Baseline characteristics of the study patients

\begin{tabular}{|c|c|c|c|}
\hline Parameters & Group $1(n=8)$ & Group $2(n=10)$ & $P$-value \\
\hline Age $(y)$, Mean \pm SD & $68.38 \pm 9.21$ & $68.80 \pm 6.74$ & 0.914 \\
\hline Time since DME diagnosis $(y)$, Mean \pm SD & $1.63 \pm 0.51$ & $2.5 \pm 1.26$ & 0.069 \\
\hline Lens status (pseudophakic/phakic ratio), \% & $50 / 50$ & $40 / 60$ & 1.000 \\
\hline \multicolumn{4}{|l|}{ Previous treatments } \\
\hline Laser/intravitreal injections, \% & $100 / 100$ & $80 / 100$ & $0.706 / 1.000$ \\
\hline Number of intravitreal injections, Mean \pm SD & $4.88 \pm 1.36$ & $8.90 \pm 2.03$ & 0.001 \\
\hline Number of anti-VEGF intravitreal injections, Mean \pm SD & $3.88 \pm 1.33$ & $7.80 \pm 1.81$ & 0.001 \\
\hline $\begin{array}{l}\text { Number of short-acting steroid (triamcinolone or dexamethasone) } \\
\text { intravitreal injections, Mean } \pm \text { SD }\end{array}$ & $1.00 \pm 0.54$ & $1.10 \pm 0.57$ & 0.693 \\
\hline Follow-up period (months), Mean \pm SD & $16.37 \pm 7.09$ & $15.00 \pm 12.94$ & 0.657 \\
\hline BCVA (ETDRS visual score), Mean \pm SD & $52.50 \pm 23.32$ & $54.70 \pm 15.68$ & 0.721 \\
\hline CMT $(\mu \mathrm{m})$, Mean \pm SD & $524.12 \pm 196.56$ & $549.00 \pm 104.29$ & 0.790 \\
\hline
\end{tabular}

Abbreviations: n, number; y, years; SD, standard deviation; \%, percentage; DME, diabetic macular edema; VEGF, vascular endothelial growth factor; BCVA, best-corrected visual acuity; ETDRS, Early Treatment of Diabetic Retinopathy Study chart; CMT, central macular thickness. $P$-value $<0.05$ is shown in bold. Note: Group $1, \leq 6$ prior intravitreal injections (early switch); Group 2, $>6$ intravitreal injections (late switch). 
Concerning OCT biomarkers at baseline, there was a higher percentage of eyes with DRIL (90.0\% versus $25.0 \%$, respectively) and EZ disruption (80.0\% versus $12.5 \%$, respectively; $)$ in group 2 than in group $1(P<0.05$ for both). The percentage of eyes with a combination of DRIL and EZ disruption was also significantly $(P<0.05)$ higher in group $2(80.0 \%)$ than in group 1 (12.5\%) (Figure 1$)$.

At the last observation point, there was a statistically significant reduction in the percentage of eyes with both DRIL and EZ disruption in group 2 than in the baseline $(12.5 \%$ versus $80.0 \%$, respectively; $P<0.05)$. The percentage of eyes with both DRIL and EZ disruption at the last observation in group 1 was the same as that observed at baseline ( $12.5 \%$ versus $12.5 \%$, respectively).

Concerning functional outcomes, BCVA at baseline was similar $(P>0.05)$ in both groups (Table 1). After intravitreal injection of an FAc implant, a similar (all $P>0.05)$ percentage of eyes achieved vision stabilization or improvement at the last observation, gained $\geq 10$ and $\geq 15$ ETDRS letters from baseline, and achieved a visual acuity of $\geq 70$ ETDRS letters (Figure 2).

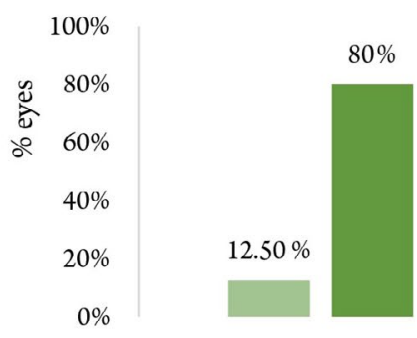

DRIL and EZ disruption

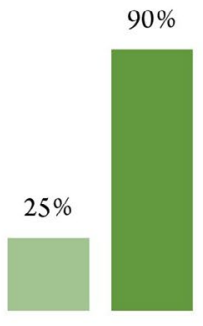

DRIL

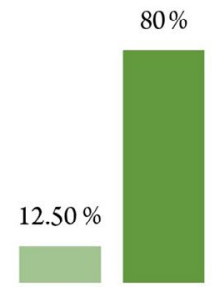

EZ disruption
Group 1

Group 2

Figure 1. Presence of disorganization of the retinal inner layers (DRIL) and/or ellipsoid zone (EZ) disruption at baseline in Groups 1 and 2. Note: Group 1, $\leq 6$ prior intravitreal injections (early switch); Group 2, $>6$ intravitreal injections (late switch).

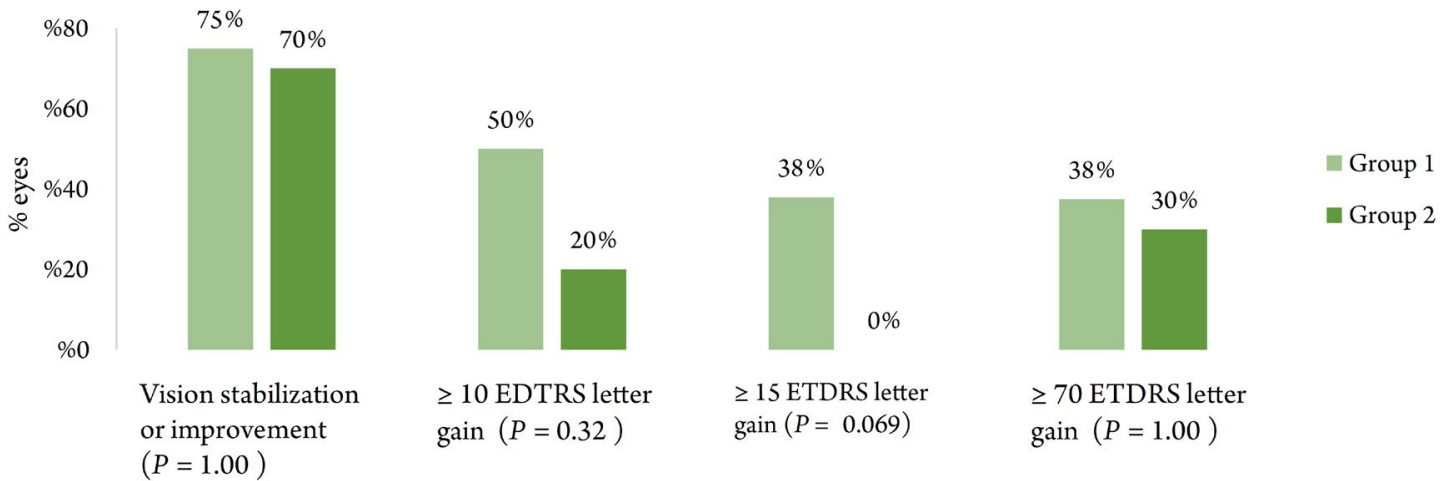

Figure 2. Visual acuity outcomes at the last observation in Groups 1 and 2. Abbreviations: ETDRS, Early Treatment Diabetic Retinopathy Study. Note: Group 1, $\leq 6$ prior intravitreal injections (early switch); Group 2, $>6$ intravitreal injections (late switch).

Table 2. Intraocular pressure events at last observation in Groups 1 and 2

\begin{tabular}{|l|l|l|l|}
\hline IOP events & Group 1 & Group 2 & \multicolumn{1}{l|}{-value } \\
\hline IOP $\geq 21 \mathbf{~ m m H g}, \mathbf{n}(\%)$ & $1(12.5)$ & $3(30.0)$ & 0.588 \\
\hline IOP-lowering medication at baseline, $\mathbf{n}(\%)$ & $3(37.5)$ & $0(0.0)$ & 0.069 \\
\hline Emer gent IOP-lowering medication at the last observation, $\mathbf{n}(\%)$ & $3(37.5)$ & $4(40.0)$ & 0.188 \\
\hline Trabeculoplasty, $\mathbf{n}(\%)$ & $0(0.0)$ & $0(0.0)$ & - \\
\hline IOP-lowering surgery, $\mathbf{n}(\%)$ & $0(0.0)$ & $0(0.0)$ & - \\
\hline
\end{tabular}

Abbreviations: IOP, intraocular pressure; n, number; \%, percentage. Note: Group 1, $\leq 6$ prior intravitreal injections (early switch); Group 2, > 6 intravitreal injections (late switch). 
At the last observation point, the mean BCVA gain was also clinically significant, with group 1 gaining +8.8 letters versus group 2 gaining +0.7 letters. Although clinically significant, no statistically significant differences were detected between the groups $(P=0.397)$.

Concerning anatomical outcomes, the mean CMT was also similar in groups 1 and 2 at baseline (Table 1). Following treatment with an FAc implant, both groups showed sustained and continuous edema resolution (i.e., $>20 \%$ reduction from baseline) over the follow-up period with similar $(P>0.05)$ mean \pm SD CMT values at the last observation in groups 1 and $2(376.88 \mu \mathrm{m} \pm 104.93$ versus $371.40 \mu \mathrm{m} \pm 183.23$, respectively). There were no statistically or clinically significant differences between the two groups $(P=0.749)$.

Concerning safety-related events, there was no significant $(P=0.242)$ change in mean IOP at the last observation in either group (Figure 3). IOP outcomes were also consistent between groups, with a similar percentage of eyes in both groups, achieving an IOP $\geq 21 \mathrm{mmHg}$ and requiring IOP-lowering drops to control pressure. Interestingly, at baseline, none of the eyes in group 2 had been treated with IOP-lowering medication versus $37.5 \%$ of the eyes in group 1 . Neither group required trabeculoplasty or IOP-lowering surgery (including trabeculectomy or any drainage devices) to manage high IOP (Table 2).
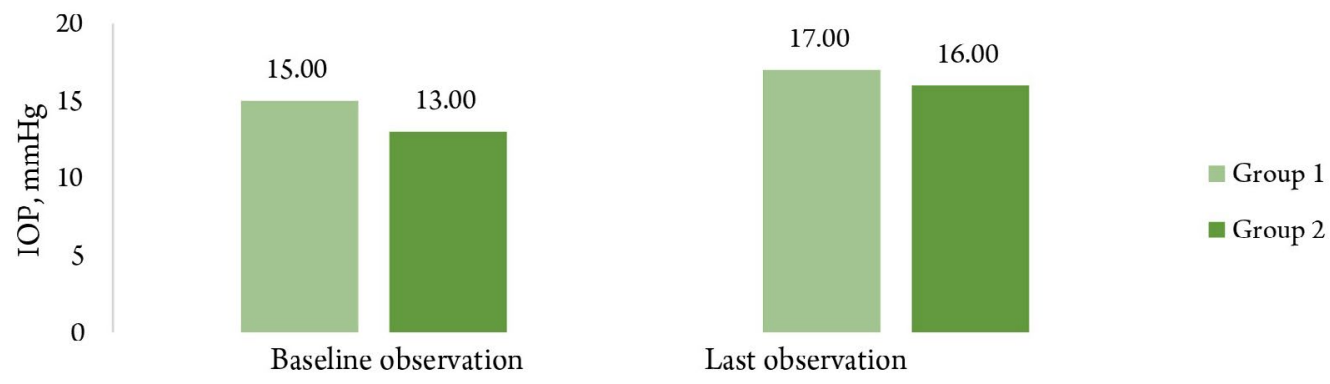

Last observation

Figure 3. Mean Intraocular pressure $(\mathrm{IOP})$ in millimeters of mercury $(\mathrm{mmHg})$ at baseline and last observation in Groups 1 and $2 . P$-value $=$ 0.242 between groups at the last observation.
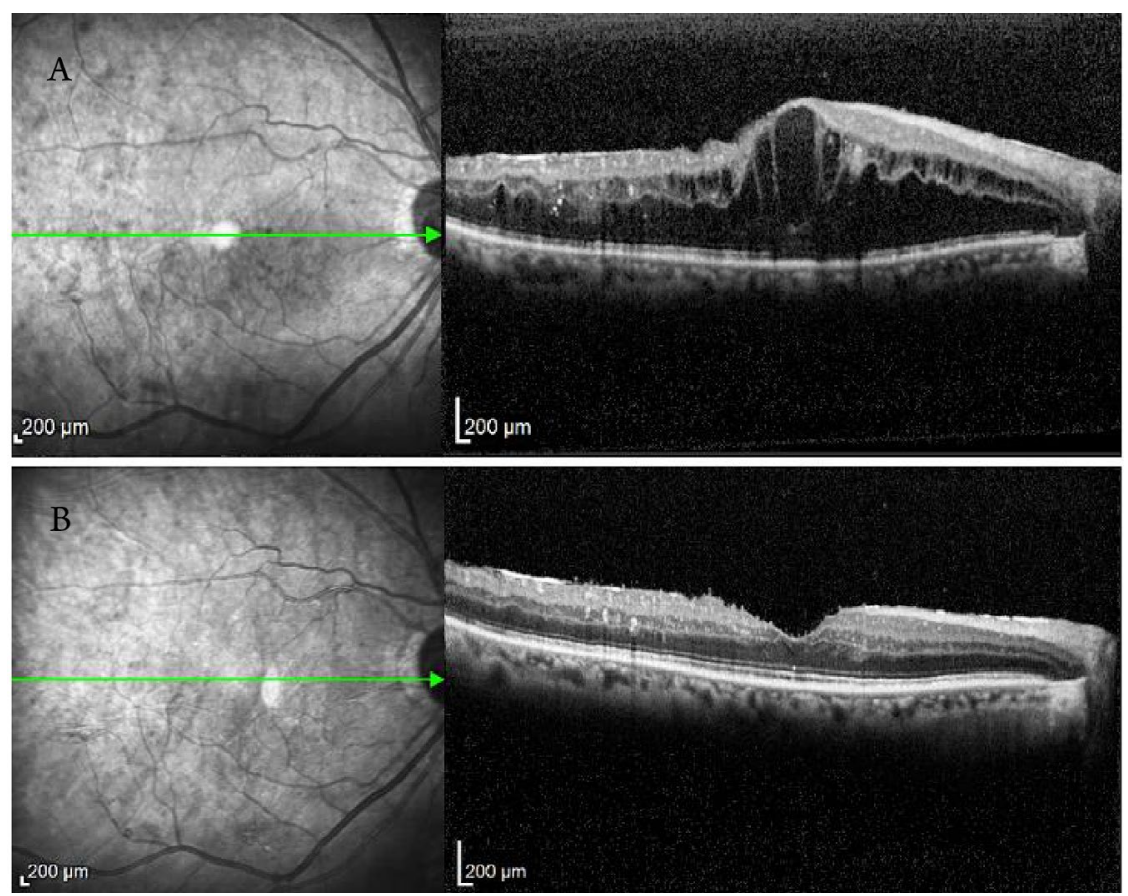

Figure 4. Two spectral-domain optical coherence tomography horizontal scans of one patient from Group 1 at baseline (A), showing disorganization of the retinal inner layers (DRIL) and ellipsoid zone (EZ) disruption in the central foveal area and at last observation (B), showing resolution of DRIL and EZ disruption. 


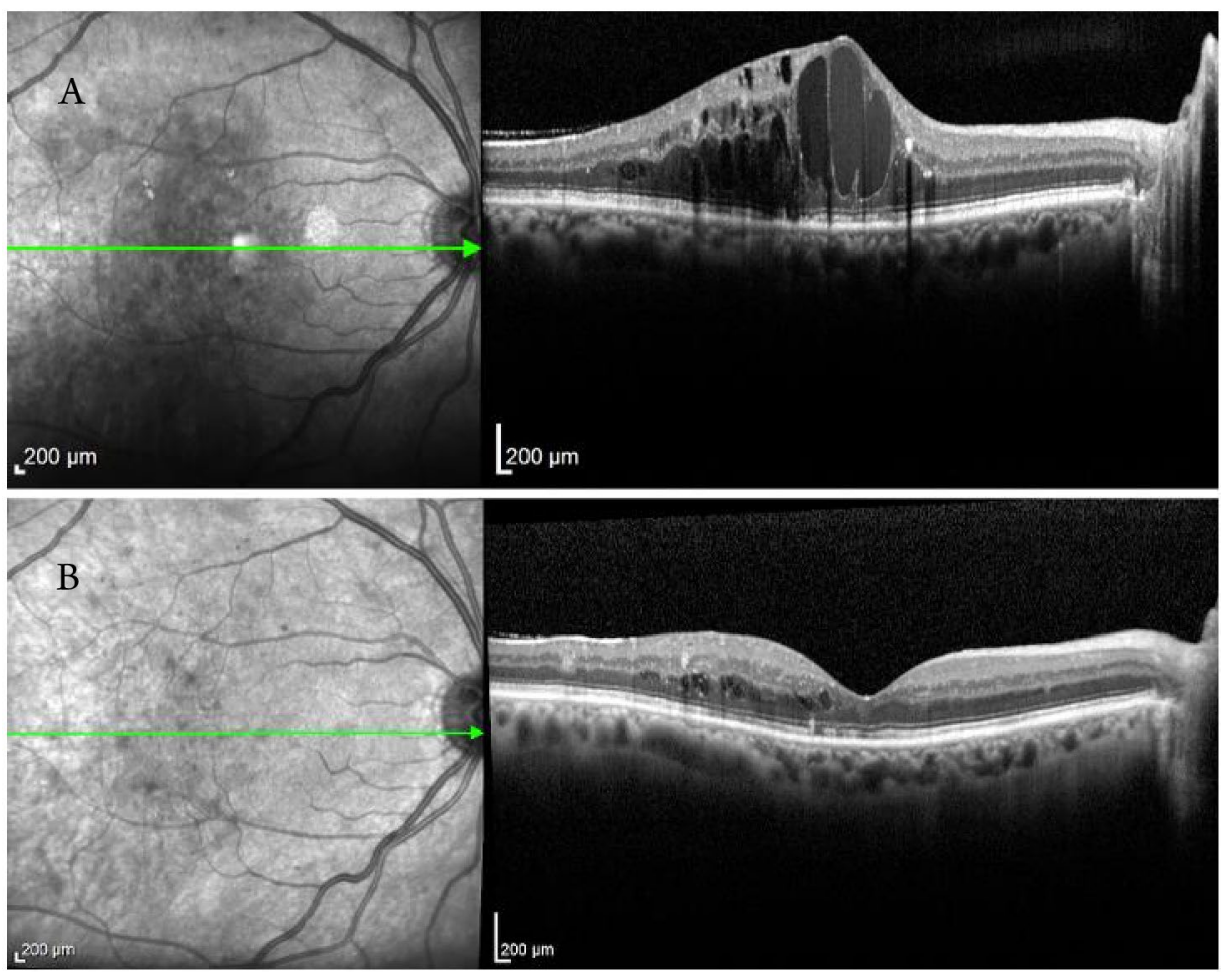

Figure 5. Two spectral-domain optical coherence tomography horizontal scans of one patient from Group 2 at baseline (A), showing disorganization of the retinal inner layers (DRIL) and ellipsoid zone (EZ) disruption in the central foveal area and at last observation (B), showing resolution of DRIL and EZ disruption.

\section{DISCUSSION}

Our study revealed that group 1 (early switch group), which had a significantly lower percentage of eyes with DRIL and EZ disruption (alone or in combination) at baseline, achieved clinically higher main visual gain at the last observation than group $2(+8.8$ letters versus +0.7 letters, respectively; $P>0.05)$. The study also showed a statistically significant difference in the evolution of OCT biomarkers between the two groups, with resolution of these biomarkers at the last observation in most eyes from group 2. There were no statistically significant differences in anatomical and safety outcomes between the two groups.

OCT technology has facilitated the identification of DRIL and EZ disruption in patients with DME, thus shedding light on the prognosis of these patients. DRIL is a noninvasive parameter that seems to be a reliable predictor of visual outcomes in eyes with $\operatorname{DME}[17,18]$. A plausible explanation for this fact is that DRIL may reflect disorganization or compromised function of some anatomical structures considered critical for the transmission of visual information, such as bipolar, Müller, and horizontal cells [14, 15, 21]. In fact, the communication pathway between photoreceptors and ganglion cells mostly depends on bipolar cell integrity $[22,23]$. When retinal thickness increases at a point capable of breaking its elastic limit caused by edema, bipolar axons may become damaged, which affects visual data signaling, ultimately leading to poor visual outcomes $[18,23]$. This is especially true for the involvement of the immediate parafoveal area and could explain the robust association between DRIL and visual acuity described in the literature $[6,14,18]$ and observed in our study. The presence of DRIL has been further considered a signal of macular capillary nonperfusion, which may also contribute to worse functional outcomes [20, 23].

The idea that chronicity plays an important role in DRIL development [18] is also reflected in our results, since group 2, which has a longer DME duration and performed a later switch to the FAc implant, had a higher percentage of eyes with DRIL (90.0\% versus $25 \% .0 \%)$, EZ disruption (80.0\% versus $12.5 \%$ ), and both DRIL and EZ disruption ( $80.0 \%$ versus $12.5 \%$ ) than group 1.

The literature suggests that the EZ layer reflects photoreceptor integrity and can also be considered an important predictor of visual prognosis in various retinal diseases [21,24-28]. Similar to DRIL, several studies have suggested that EZ integrity is related to better visual outcomes after anti-VEGF and dexamethasone implant treatment $[6,14,21]$. Our study showed the same benefits after treatment with an FAc implant. It has 
been suggested that DRIL is strongly related to disruption of the EZ layer and external limiting membrane integrity [15]. Our work confirms this relationship, since the majority of eyes with DRIL had EZ disruption. Maheshwary et al. concluded that the disruption of the photoreceptor inner and outer segments, as well as the percentage of this disruption, are both essential visual prognosticators in patients with DME [16]. It is possible that the mechanisms responsible for inner retinal layer disorganization may also play a role in concomitant disruption of the outer retinal anatomy [15].

In the present study, patients from group 1 achieved better functional outcomes. Although clinically significant, no statistically significant differences were detected between the groups $(+8.8$ letters versus +0.7 letters). These results can be explained by three reasons. First, since those eyes switched earlier to FAc implant treatment, they had shorter DME duration and consequently shorter exposure to the swelling effect on retinal anatomy integrity [28]. Second, those eyes had a lower percentage of DRIL and/or EZ disruption at baseline $[14,15]$. Third, those eyes were also less exposed to the effect of consecutive, recurrent short-acting intravitreal treatments $[2,7]$. The number of previous intravitreal injections was statistically different between the two groups, reflecting a possible association between these biomarkers and intravitreal injection number, supporting this hypothesis.

However, a small percentage of eyes achieving vision stability was also observed in group 2 . The fact that DRIL may gradually regress after DME resolution, as observed in our patients (Figures 4 and 5), could explain these results $[18,21]$. Indeed, there was a statistically significant difference in the evolution of prognostic biomarkers between the two groups, supporting the idea that FAc implants may improve retinal integrity. This finding also suggests that even eyes with a higher percentage of DRIL and EZ disruption may benefit, from an anatomical and visual viewpoint, from switching to FAc implants.

The functional effectiveness outcomes obtained here are in agreement with the results of current reports and other studies of FAc implants for the treatment of recurrent and/or persistent DME in a real-world setting $[7,8]$. In this regard, according to the ILUVIEN Registry Safety Study (IRISS), patients with short-term and long-term DME gained approximately nine letters and one letter, respectively, at month 15 , which is consistent with our findings [19]. All these results support the idea that switching to an FAc implant as soon as an insufficient response to previous treatment is detected could have more functional advantages.

In our study, the early or late switch to the FAc implant did not have an impact on the anatomical results. Structurally, the mean reduction in CMT was similar in both groups, which showed sustained and continuous edema resolution, in both cases exceeding $20 \%$ reduction from the baseline value. In relation to safety outcomes, there was no significant change in mean IOP at the last observation in either group. IOP outcomes were also consistent in the two groups, with a similar percentage of eyes achieving an IOP $\geq 21 \mathrm{mmHg}$ and requiring IOP-lowering drops to control pressure.

The major limitations of this study are its retrospective nature and the limited number of treated eyes. However, to reduce or eliminate some of the data acquisition inconsistencies, the present study included methodological approaches, such as extended electronic medical data reports, certified ETDRS visual acuity measures, and SD-OCT image acquisition following the protocol, thus maximizing the accuracy of clinical data. Although prospective and randomized studies, including a larger patient cohort, are warranted to obtain further conclusions. The analysis of morphological features and biomarkers on SD-OCT scans is expected to have a growing importance in managing treatment and visual outcome expectations in patients with DME.

\section{CONCLUSIONS}

This study showed the benefit of switching to an FAc implant in patients with chronic DME after an insufficient response to other intravitreal treatments, especially if done early. OCT biomarkers, namely DRIL and/or EZ disruption, are useful prognostic predictors, particularly in DME management.

\section{ETHICS DECLARATIONS}

Ethical approval: The study protocol was approved by the institutional ethics committee (Health Ethical Committee of Beatriz Angelo Hospital). Written informed consent was obtained from all patients.

Conflicts of interests: None.

\section{FUNDING}

\section{None.}




\section{ACINOWLEDGMENT}

\section{None.}

\section{REFERENCES}

1. Campos A, Campos EJ, do Carmo A, Caramelo F, Martins J, Sousa JP, et al. Evaluation of markers of outcome in real-world treatment of diabetic macular edema. Eye Vis (Lond). 2018;5:27. doi: 10.1186/s40662-018-0119-9 pmid: 30386806

2. Muftuoglu IK, Mendoza N, Gaber R, Alam M, You Q, Freeman WR. Integrity of Outer Retinal Layers after Resolution of Central Involved Diabetic Macular Edema. Retina. 2017;37(11):2015-2024. doi: 10.1097/IAE.0000000000001459 pmid: 28092342

3. Coelho J, Malheiro L, Melo Beirao J, Meireles A, Pessoa B. Real-world retrospective comparison of $0.19 \mathrm{mg}$ fluocinolone acetonide and $0.7 \mathrm{mg}$ dexamethasone intravitreal implants for the treatment of diabetic macular edema in vitrectomized eyes. Clin Ophthalmol. 2019;13:1751-1759. doi: 10.2147/OPTH.S201611 pmid: 31571814

4. Vujosevic S, Torresin T, Bini S, Convento E, Pilotto E, Parrozzani R, et al. Imaging retinal inflammatory biomarkers after intravitreal steroid and anti-VEGF treatment in diabetic macular oedema. Acta Ophthalmol. 2017;95(5):464-471. doi: 10.1111/aos.13294 pmid: 27775223

5. Bandello F, Berchicci L, La Spina C, Battaglia Parodi M, Iacono P. Evidence for anti-VEGF treatment of diabetic macular edema. Ophthalmic Res. 2012;48 Suppl 1:16-20. doi: 10.1159/000339843 pmid: 22907145

6. Cavalleri M, Cicinelli MV, Parravano M, Varano M, De Geronimo D, Sacconi R, et al. Prognostic role of optical coherence tomography after switch to dexamethasone in diabetic macular edema. Acta Diabetol. 2020;57(2):163-171. doi: 10.1007/s00592-01901389-4 pmid: 31375899

7. Kodjikian L, Baillif S, Creuzot-Garcher C, Delyfer MN, Matonti F, Weber M, et al. Real-World Efficacy and Safety of Fluocinolone Acetonide Implant for Diabetic Macular Edema: A Systematic Review. Pharmaceutics. 2021;13(1):72. doi: 10.3390/pharmaceutics13010072 pmid: 33430389

8. Rehak M, Busch C, Unterlauft JD, Jochmann C, Wiedemann P. Outcomes in diabetic macular edema switched directly or after a dexamethasone implant to a fluocinolone acetonide intravitreal implant following anti-VEGF treatment. Acta Diabetol. 2020;57(4):469-478. doi: 10.1007/s00592-019-01439-x pmid: 31749051

9. Cicinelli MV, Rabiolo A, Zollet P, Capone L, Lattanzio R, Bandello F. Persistent or Recurrent Diabetic Macular Edema After Fluocinolone Acetonide 0.19 mg Implant: Risk Factors and Management. Am J Ophthalmol. 2020;215:14-24. doi: 10.1016/j. ajo.2020.03.016 pmid: 32209341

10. Diabetic Retinopathy Clinical Research N, Wells JA, Glassman AR, Ayala AR, Jampol LM, Aiello LP, et al. Aflibercept, bevacizumab, or ranibizumab for diabetic macular edema. N Engl J Med. 2015;372(13):1193-203. doi: 10.1056/NEJMoa1414264 pmid: 25692915

11. Gonzalez VH, Campbell J, Holekamp NM, Kiss S, Loewenstein A, Augustin AJ, et al. Early and Long-Term Responses to Anti-Vascular Endothelial Growth Factor Therapy in Diabetic Macular Edema: Analysis of Protocol I Data. Am J Ophthalmol. 2016;172:7279. doi: 10.1016/j.ajo.2016.09.012 pmid: 27644589

12. Campochiaro PA, Nguyen QD, Hafiz G, Bloom S, Brown DM, Busquets M, et al. Aqueous levels of fluocinolone acetonide after administration of fluocinolone acetonide inserts or fluocinolone acetonide implants. Ophthalmology. 2013;120(3):583-587. doi: 10.1016/j.ophtha.2012.09.014 pmid: 23218184

13. Arrigo A, Capone L, Lattanzio R, Aragona E, Zollet P, Bandello F. Optical Coherence Tomography Biomarkers of Inflammation in Diabetic Macular Edema Treated by Fluocinolone Acetonide Intravitreal Drug-Delivery System Implant. Ophthalmol Ther. 2020;9(4):971-980. doi: 10.1007/s40123-020-00297-z pmid: 32914324

14. Sun JK, Radwan SH, Soliman AZ, Lammer J, Lin MM, Prager SG, et al. Neural Retinal Disorganization as a Robust Marker of Visual Acuity in Current and Resolved Diabetic Macular Edema. Diabetes. 2015;64(7):2560-70. doi: 10.2337/db14-0782 pmid: 25633419

15. Sun JK, Lin MM, Lammer J, Prager S, Sarangi R, Silva PS, et al. Disorganization of the retinal inner layers as a predictor of visual acuity in eyes with center-involved diabetic macular edema. JAMA Ophthalmol. 2014;132(11):1309-16. doi: 10.1001/jamaophthalmol.2014.2350 pmid: 25058813

16. Maheshwary AS, Oster SF, Yuson RM, Cheng L, Mojana F, Freeman WR. The association between percent disruption of the photoreceptor inner segment-outer segment junction and visual acuity in diabetic macular edema. Am J Ophthalmol. 2010;150(1):63-67 e1. doi: 10.1016/j.ajo.2010.01.039 pmid: 20451897

17. Markan A, Agarwal A, Arora A, Bazgain K, Rana V, Gupta V. Novel imaging biomarkers in diabetic retinopathy and diabetic macular edema. Ther Adv Ophthalmol. 2020;12:2515841420950513. doi: 10.1177/2515841420950513 pmid: 32954207

18. Stuart A, Shah GK, Singh R, Wykoff CC (2017). 'DRIL a Robust Biomarker for DME'. [Online]. Available at:https://www.aao.org/ eyenet/article/dril-a-robust-biomarker-for-dme. (Accessed: April 19, 2021).

19. Chakravarthy U, Taylor SR, Koch FHJ, Castro de Sousa JP, Bailey C, Group IRSSI. Changes in intraocular pressure after intravitreal fluocinolone acetonide (ILUVIEN): real-world experience in three European countries. Br J Ophthalmol. 2019;103(8):1072-1077. doi: 10.1136/bjophthalmol-2018-312284 pmid: 30242062

20. Rosser DA, Cousens SN, Murdoch IE, Fitzke FW, Laidlaw DA. How sensitive to clinical change are ETDRS logMAR visual acuity measurements? Invest Ophthalmol Vis Sci. 2003;44(8):3278-81. doi: 10.1167/iovs.02-1100 pmid: 12882770

21. Eraslan S, Yildirim O, Dursun O, Dinc E, Orekici Temel G. Relationship Between Final Visual Acuity and Optical Coherence Tomography Findings in Patients with Diabetic Macular Edema Undergoing Anti-VEGF Therapy. Turk J Ophthalmol. 2020;50(3):163-168. doi: 10.4274/tjo.galenos.2019.91962 pmid: 32631004

22. Bringmann A, Unterlauft JD, Wiedemann R, Barth T, Rehak M, Wiedemann P. Two different populations of Muller cells stabilize the structure of the fovea: an optical coherence tomography study. Int Ophthalmol. 2020;40(11):2931-2948. doi: 10.1007/s10792020-01477-3 pmid: 32632619

23. Pelosini L, Hull CC, Boyce JF, McHugh D, Stanford MR, Marshall J. Optical coherence tomography may be used to predict visual acuity in patients with macular edema. Invest Ophthalmol Vis Sci. 2011;52(5):2741-8. doi: 10.1167/iovs.09-4493 pmid: 20538987

24. Nicholson L, Ramu J, Triantafyllopoulou I, Patrao NV, Comyn O, Hykin P, et al. Diagnostic accuracy of disorganization of the retinal inner layers in detecting macular capillary non-perfusion in diabetic retinopathy. Clin Exp Ophthalmol. 2015;43(8):735-41. doi: $10.1111 /$ ceo. 12557 pmid: 25998983

25. Ito S, Miyamoto N, Ishida K, Kurimoto Y. Association between external limiting membrane status and visual acuity in diabetic macular oedema. Br J Ophthalmol. 2013;97(2):228-32. doi: 10.1136/bjophthalmol-2011-301418 pmid: 23172879 
26. Forooghian F, Stetson PF, Meyer SA, Chew EY, Wong WT, Cukras C, et al. Relationship between photoreceptor outer segment length and visual acuity in diabetic macular edema. Retina. 2010;30(1):63-70. doi: 10.1097/IAE.0b013e3181bd2c5a pmid: 19952996

27. Murakami T, Nishijima K, Sakamoto A, Ota M, Horii T, Yoshimura N. Association of pathomorphology, photoreceptor status, and retinal thickness with visual acuity in diabetic retinopathy. Am J Ophthalmol. 2011;151(2):310-7. doi: 10.1016/j.ajo.2010.08.022 pmid: 21145531

28. Sadda SR, Campbell J, Dugel PU, Holekamp NM, Kiss S, Loewenstein A, et al. Relationship between duration and extent of oedema and visual acuity outcome with ranibizumab in diabetic macular oedema: A post hoc analysis of Protocol I data. Eye (Lond). 2020;34(3):480-490. doi: 10.1038/s41433-019-0522-z pmid: 31320738 